\title{
Super-resolution vibrational imaging using expansion stimulated Raman scattering microscopy
}

\author{
Lixue Shi ${ }^{1}$, Aleksandra Klimas ${ }^{2}$, Brendan Gallagher ${ }^{2}$, Zhangyu Cheng $^{2}$, Feifei Fu ${ }^{2}$, Piyumi Wijesekara ${ }^{3}$, \\ Yupeng $\mathrm{Miao}^{1}, \mathrm{Xi} \mathrm{Ren}^{3}$, Yongxin Zhao ${ }^{2 *}$, Wei Min ${ }^{1,4^{*}}$ \\ ${ }^{1}$ Department of Chemistry, Columbia University, New York, NY, USA. \\ ${ }^{2}$ Department of Biological Sciences, Carnegie Mellon University, Pittsburgh, PA, USA. \\ ${ }^{3}$ Department of Biomedical Engineering, Carnegie Mellon University, Pittsburgh, PA, USA. \\ ${ }^{4}$ Kavli Institute for Brain Science, Columbia University, New York, NY, USA. \\ *Corresponding author: wm2256@columbia.edu,yongxinz@andrew.cmu.edu
}

\begin{abstract}
Stimulated Raman scattering (SRS) microscopy is an emerging technology that provides high chemical specificity for endogenous biomolecules and can circumvent common constraints of fluorescence microscopy including limited capabilities to probe small biomolecules and difficulty resolving many colors simultaneously due to spectral overlap. However, the resolution of SRS microscopy remains governed by the diffraction limit. To overcome this, we describe a new technique called Molecule Anchorable Gel-enabled Nanoscale Imaging of Fluorescence and stImulatEd Raman Scattering microscopy (MAGNIFIERS), that integrates SRS microscopy with expansion microscopy (ExM). ExM is a powerful strategy providing significant improvement in imaging resolution by physical magnification of hydrogel-embedded preserved biological specimens. MAGNIFIERS offers chemical-specific nanoscale imaging with sub-50 nm resolution and has scalable multiplexity when combined with multiplex Raman probes and fluorescent labels. We used MAGNIFIERS to visualize nanoscale features in a label-free manner with $\mathrm{C}-\mathrm{H}$ vibration of proteins, lipids and DNA in a broad range of biological specimens, from mouse brain, liver and kidney to human lung organoid. In addition, we applied MAGNIFIERS to track nanoscale features of protein synthesis in protein aggregates using metabolic labeling of small metabolites. Finally, we used MAGNIFIERS to demonstrate 8-color nanoscale imaging in an expanded mouse brain section. Overall, MAGNIFIERS is a valuable platform for super-resolution label-free chemical imaging, high-resolution metabolic imaging, and highly multiplexed nanoscale imaging, thus bringing SRS to nanoscopy.
\end{abstract}




\section{Introduction}

While fluorescence microscopy has been the predominant imaging modality in biophotonics, it faces fundamental limitations for many advanced imaging applications. Going beyond fluorescence, stimulated Raman scattering (SRS) microscopy is emerging as a powerful modality for illuminating otherwise invisible chemical bonds of biomolecules in complex biological contexts ${ }^{1,2}$. In contrast to fluorescence imaging, SRS generates high chemical specificity via probing vibrational transitions, enabling label-free detection of vital biomolecules of proteins, lipids, nucleic acids, etc ${ }^{1,3}$. Moreover, integration of SRS with vibrational tags renders a paradigm of bioorthogonal chemical imaging for studying metabolic activities in cells and tissues ${ }^{2,4,5}$. The tiny size and good biocompatibility of vibrational tags, such as stable isotopes and triple bonds, enable non-perturbative tracing of small molecules ${ }^{4}$, which is difficult to achieve with fluorescence because of the bulky fluorophores. Furthermore, by virtue of much narrower vibrational peaks $\left(\sim 10 \mathrm{~cm}^{-1}\right)$ compared to fluorescence peaks $\left(\sim 500 \mathrm{~cm}^{-1}\right)$, Raman imaging offers scalable multiplexity and breaks the 'palette number barrier' of fluorescence, which can typically only image 4-5 colors simultaneously due to the overlap of inherently broad fluorescence spectra. In this regard, development of rainbow-like Raman dyes leverages the sensitivity of SRS and opens the door for supermultiplexed vibrational imaging ${ }^{6-8}$. In particular, by combining electronic pre-resonance spectroscopy with stimulated Raman scattering (SRS) microscopy (i.e., epr-SRS), the Raman cross sections of electronically coupled vibrational modes in light-absorbing dyes can be enhanced by $10^{13}$-fold ${ }^{6,9}$. Owing to this drastic enhancement, we achieved nanomolar sensitivity of Raman-active dyes (such as commercial far-red fluorescent dyes and specially-designed MARS probes), and demonstrated epr-SRS imaging of specific proteins $6,10,11$.

Collectively, SRS imaging circumvents the fundamental bottlenecks of fluorescence microscopy and provides unique benefits including label-free chemical imaging of endogenous biomolecules ${ }^{1,} 12$, nonperturbative mapping of small metabolites ${ }^{4}$, and simultaneous profiling of a large group of specific biomarkers $^{6,11}$. Yet, being an optical technique that uses near infrared or visible light, SRS microscopy is still constrained to diffraction-limited spatial resolution $(\sim 300 \mathrm{~nm})$. Thus, many crucial nanoscale organizations in cells and tissues cannot be resolved by the current iteration of SRS microscopy. Despite extensive efforts in extending Raman imaging beyond the diffraction limit, sophisticated instrumentation is required and only moderate resolution improvement (typically less than 2-fold) has been achieved, resulting in limited utilization in biological specimens ${ }^{13-18}$. Hence, vibrational-based nanoscale imaging approaches are still underexplored territory in biomedical research.

Over the last half decade, a sample-oriented nanoscale imaging technique known as expansion microscopy (ExM) has emerged as a non-optical means of achieving effective resolutions beyond the diffraction limit. ExM is a powerful technique for nanoscale optical imaging through physical magnification of tissue samples embedded in a water-swellable polymer gel ${ }^{19}$. ExM was initially developed for fluorescence microscopy with a $\sim 4.5 \times$ expansion in each dimension in pure water and quickly evolved to different variants with widespread use in biology and pathology ${ }^{20-24}$. Conceptually, the principle of ExM could be applied to overcome the diffraction limit of SRS imaging. However, common ExM variants ${ }^{22,23,25}$ rely on strong protease digestion to facilitate expansion, thereby destroying endogenous proteins. On the other hand, ExM variants that retain proteins after expansion ${ }^{26-28}$ are more compatible with SRS imaging, but they require special fixation protocols and are only validated on culture cells and mouse brain tissues. A very recent technique called VISTA was developed for label-free chemical imaging of proteins on expanded mouse brain tissues using a protein-retention ExM variant ${ }^{29}$, but it inherits the critical limitation of this ExM variant on tissue compatibility and resolution gain. 
Recently, a new ExM framework has emerged that offers larger expansion factors along with broad biomolecule retention and tissue compatibility ${ }^{30}$, capable of expanding a multitude of tissue types with standard fixation, from PFA-fixed mouse brain and human lung organoid to an array of clinically relevant formalin-fixed paraffin-embedded (FFPE) human tissues which have proved challenging to expand in the past. By combining SRS imaging with this framework, we lay out an approach named Molecule Anchorable Gel-enabled Nanoscale Imaging of Fluorescence and stimulatEd $\underline{\text { Raman }}$ Scattering microscopy (MAGNIFIERS). Harnessing the superior retention of biomolecular contents and general sample compatibility across multiple tissue types and fixation methods of the new ExM framework, the MAGNIFIERS approach provides excellent reversible linear expansion up to $~ 7.2$-fold providing lateral resolutions of $\sim 41 \mathrm{~nm}(298 \mathrm{~nm} / 7.2=41.4 \mathrm{~nm})$ and axial resolutions of $\sim 194 \mathrm{~nm}(1400 \mathrm{~nm} / 7.2=194 \mathrm{~nm})$, with great flexibility to couple vibrational metabolic labeling and Raman dye staining.

MAGNIFIERS revitalizes a full spectrum of utilities of SRS imaging into the sub-diffraction regime (Fig. 1a). Specifically, we show that MAGNIFIERS supports label-free nanoscale imaging of endogenous biomolecules including proteins, lipids, and DNA. We further demonstrate its power for high-resolution metabolic imaging of protein aggregates in cells labeled with deuterium-substituted amino acids. Finally, we use MAGNIFIERS to map the nanoscale locations of 8 different markers in mouse brain tissue in one shot by applying multiplex Raman labels and conventional fluorescent probes.

\section{Results}

\section{Integration of SRS with an optimized biomolecule-retention ExM protocol (MAGNIFY).}

To fully demonstrate the potential of expansion SRS imaging, we chose a new ExM strategy that achieves a favorable balance between biomolecule retention, expansion factor, and sample generality ${ }^{30}$. The new method is called Molecule Anchorable Gel-enabled Nanoscale In-situ Fluorescence MicroscopY $(\mathrm{MAGNIFY})^{30}$. To demonstrate its utility for SRS imaging, we first quantified the protein retention rate by imaging $\mathrm{CH}_{3}$ stretching vibration of proteins at $2941 \mathrm{~cm}^{-1}$ in paraformaldehyde (PFA)-fixed mouse kidney tissue pre- and post-expansion (Fig. 1b). Remarkably, we found a near $100 \%$ protein retention rate using the MAGNIFY approach (Fig. 1b-c), substantially higher than that of protein-retention expansion microscopy using 6-((acryloyl)amine) hexanoic acid (AcX) as an anchoring agent (i.e. proExM ${ }^{22}$, Fig. 1d and Supplementary Fig.1), ensuring optimal sensitivity and effective resolution. The reasons for the optimized biomolecule retention rate are twofold. First, methacrolein was employed as part of the monomer solution to efficiently attach biomolecules to the growing matrix during the in situ polymerization step, eliminating the need of stepwise reactions of anchoring and polymerization (Fig. 1a). Second, rather than strong protease digestion, the gel-embedded specimens were treated with a denaturantrich solution in $80-90^{\circ} \mathrm{C}$ for homogenization to ensure maximum retention of biomolecules in the gel.

MAGNIFIERS relied on a reformulated hydrogel (Fig. 1a) which allows isotropic physical expansion up to 10 -fold in a single round ${ }^{30}$. To theoretically estimate the improved spatial precision, we calibrated the resolution of the current SRS microscopy by imaging single $100-\mathrm{nm}$ polystyrene beads at $3056 \mathrm{~cm}^{-1}$ (Supplementary Fig. 2). Cross-section profiles fitted by the Gaussian function indicated the full width at half maximum (FWHM) of lateral direction of $298 \mathrm{~nm}$ (Supplementary Fig. 2a). Thus, effective resolution of $\sim 30 \mathrm{~nm}$ is possible given an expansion factor (EF) of around 10 (Fig. 1e). Unless stated otherwise, the reformulated hydrogel was used for expanding all biological specimens.

MAGNIFIERS is broadly applicable to a wide range of specimens (Supplementary Fig. 3), as it is compatible with common fixation methods and multiple tissue types, including mechanically tough tissues such as kidney and liver. For instance, SRS imaging of $\mathrm{CH}_{3}$ stretching at $2941 \mathrm{~cm}^{-1}$ visualized rich three- 
dimensional (3D) structural information in cultured cells and mouse tissues such as the brain, liver, kidney, and pancreas (Fig. 1f, Supplementary Video 1), thanks to the intrinsic 3D sectioning capability from the two-photon excitation nature of SRS. In addition, MAGNIFIERS is suitable for human organoids (Supplementary Fig. 3), a favorable model system for human biology and medicine. Going beyond freshly preserved cells and tissues, archival samples such as FFPE sections of human brain, kidney and spleen, which is a popular form of preparation for biopsy specimens that aids in clinical examination and pathology research, are also compatible with MAGNIFIERS (Fig. 1f, Supplementary Fig. 3).

\section{Label-free visualization of biological and pathological ultrastructures.}

Encouraged by the near $100 \%$ protein retention, we envision it is advantageous for unveiling nanoscale features without the need to label or stain with fluorescent dyes. In standard SRS microscopy, C-H stretching region at $\sim 2800-3050 \mathrm{~cm}^{-1}$ is desirable to image endogenous species ${ }^{3}$. However, the expanded sample might exhibit O-H background $\left(\sim 2900-3700 \mathrm{~cm}^{-1}\right)$ from the water (typically $>90 \%$ by volume) in the sample and $\mathrm{C}-\mathrm{H}$ background from the gel matrix itself, thus potentially overwhelming the signal of interest. To study this, we performed hyperspectral SRS analysis of expanded FFPE human kidney tissues (Fig. 2a, Supplementary Fig. 4a). We chose FFPE tissue as a test because of its simple Raman spectrum, as protein is the major component and most lipids are removed in deparaffinization before expansion (Supplementary Fig. 4b). By analyzing the spectra of a tissue region and a blank gel region (Fig. 2a, left), we observed strong $\mathrm{C}-\mathrm{H}$ signals on the tissue area but only a background from the tail of water's O-H vibration in the blank gel region (Fig. 2a, right). The absence of $\mathrm{C}-\mathrm{H}$ peaks in the blank gel suggests that the $\mathrm{C}-\mathrm{H}$ signal from the gel matrix is actually negligible, likely due to the low concentration of the hydrogel. After subtracting the water background, which is broad and smooth, the spectral shape recapitulates a typical $\mathrm{CH}_{3}$ mode of proteins with a major peak at $\sim 2941 \mathrm{~cm}^{-1}$ (Fig. 2b), consistent with the fact that proteins are the major anchored biomolecules in FFPE tissue. Based on our spectral analyses, we confirmed that the polymer gel is "Raman transparent" in the C-H region and that the leak of the O-H background can be readily subtracted, validating using the $\mathrm{CH}_{3}$ vibration at $2941 \mathrm{~cm}^{-1}$ for imaging total proteins in expanded specimens.

It is valuable to compare the protein distribution pattern from SRS imaging of $\mathrm{CH}_{3}$ vibration at 2941 $\mathrm{cm}^{-1}$ to that from fluorescence imaging of stained total protein content ${ }^{31,32}$. By performing SRS and fluorescence imaging on an expanded mouse brain section stained with N-hydroxysuccidimidyl (NHS) ester-dye conjugates, we observed similar patterns (Fig. 2c). This morphological agreement between SRS and fluorescence images corroborates our prior spectral analysis.

The effective resolution is jointly determined by the objective's numerical aperture (NA) and the achievable EF, which can be controlled by osmotic pressure of the imaging solution (Fig. 1e). Given a practical EF of 7.2 with good signal (Fig. 2d), an effective resolution of $\sim 298 / 7.2=41 \mathrm{~nm}$ is feasible. With this improved resolution, we resolved sub-diffraction-limited dots on expanded mouse brain tissue, proved by a fitted FWHM of $104 \mathrm{~nm}$ (Fig. 2e).

MAGNIFIERS can unveil a broad range of biological or pathological ultrastructure features solely via label-free protein imaging. First, the microscopic view of blood-brain barrier in mouse brain tissue, including single endothelial cells and a pericyte, was visualized (Fig. 2f), supported by the co-staining with lectins as a blood vessel marker (Fig. 2g, Supplementary Fig. 4c). Benefiting from good sample compatibility of MAGNIFY, we further revealed interesting nanoscale architectures in both PFA-fixed mouse kidney and FFPE human kidney specimens. For instance, cilia were visualized at the inner layer of the proximal convoluted tubule (PCT) in mouse kidney (Fig. 2h). In addition, we observed sub- 
diffraction-limited structures of interdigitated foot processes of podocytes in the glomeruli of human (Fig. 2i) and mouse kidney tissues (Supplementary Fig. 5a-e), which is a clinical metric for many nephrotic kidney diseases such as kidney minimal change disease ${ }^{33}$. Moreover, in expanded human bronchial epithelial cells-derived lung organoids, flower-like cilia architectures were faithfully elucidated, projecting from the apical epithelial surface (Fig. 2j-k, Supplementary Fig. 5f, Supplementary Video 2). Intracellular structures and patterns of surrounding apoptotic bodies were also clearly observed (Supplementary Fig. 5g-h). Remarkably, at the basal foot of cilia, the ring structure of ciliary base bodies, which are composed of a 9+0 microtubule arrangement and have an inner diameter of around $200 \mathrm{~nm}^{34}$, were precisely identified (Fig. 21-m, yellow arrows).

\section{Label-free nanoscale imaging of chemical compositions.}

The MAGNIFY framework is known to retain biomolecules other than proteins such as lipids and nucleic acids ${ }^{30}$. Thus, MAGNIFIERS holds great promise for label-free chemically-defined nanoscale imaging in a comprehensive view. Yet, the uncharacterized issue is whether SRS can detect these molecules with sufficient contrast. Prior SRS spectral studies have dissected the C-H signals into contributions from proteins $\left(\sim 2931 \mathrm{~cm}^{-1}\right)$, lipids $\left(\sim 2854 \mathrm{~cm}^{-1}\right)$ and DNA $\left(\sim 2956 \mathrm{~cm}^{-1}\right)$ through linear spectral unmixing ${ }^{12}$. We started by applying this strategy to analyze expanded human lung organoids with an EF of 4.5. In the unmixed images which are labeled as $\mathrm{CH}_{\mathrm{Pr}}, \mathrm{CH}_{\mathrm{L}}$ and $\mathrm{CH}_{\mathrm{DNA}}$ (Fig. 3a-b), we observed the presence of lipids in small-size extracellular vesicles ${ }^{35}$ (EVs, secreted membrane vesicles), some of which also contain $\mathrm{CH}_{\text {DNA }}$ signals. We next performed a hyperspectral analysis of the entire $\mathrm{C}$ - $\mathrm{H}$ region (Supplementary Fig. 6a). By examining the pixel-averaged SRS spectrum of all small EVs (i.e. exosomes) in the region of interest, a distinct side peak around $2865 \mathrm{~cm}^{-1}$ appeared (Fig. 3c, Supplementary Fig. 6b), in agreement with the peak signature of $\mathrm{CH}_{2}$ vibration from lipids. Exosomes contain high levels of cholesterol, sphingomyelin and ceramide, similar to the composition of detergent-resistant lipid rafts ${ }^{36}$. As a control, the SRS spectrum of the cell predominantly reflects the chemical footprint of proteins (Fig. 3c, blue). Furthermore, we generated the Raman spectrum of a single DNA-containing EV (pink arrowed in Fig. 3b) which displayed another pronounced shoulder peak of $\sim 2955 \mathrm{~cm}^{-1}$ originating from DNA as we anticipated (Fig. 3d, Supplementary Fig. 6c). This observation is in line with prior reports demonstrating that EVs contain proteins, lipids and nucleic acids ${ }^{35,37}$.

EVs have been growingly recognized to play crucial roles in diverse biological systems to shuttle molecules across cells, showing promise to aid in disease diagnosis or to be engineered to deliver therapeutic payloads ${ }^{38}$. However, imaging EVs at the single-vesicle level remains a challenging task due to their small size and potential labeling bias ${ }^{39}$. Here, we have demonstrated that MAGNIFIERS enables analyzing chemical compositions of single exosomes in their spatial context without labeling (Fig. 3a-d). It is noteworthy that we only observed lipids in small EVs at submicron size, but not others such as large microvesicles and apoptotic bodies (Supplementary Fig. 6d). This could be explained by the differential lipid compositions of EVs determined by their biogenesis processes ${ }^{35}$. In this regard, the existence of $\mathrm{CH}_{\mathrm{L}}$ signals may work as a marker to identify EV biogenesis.

We also observed interesting patterns from the $\mathrm{CH}_{\mathrm{L}}$ channel in the expanded PFA-fixed mouse brain under varied expansion levels of up to 4.5 -fold (Supplementary Fig. 7). For instance, by looking at the white matter in cerebellum, astounding lipid architectures were captured at the edge of myelin fibers which could be visualized by the protein channel (Supplementary Fig. 7a). Transverse cross-section of myelinated axons was clearly unveiled in the $\mathrm{CH}_{\mathrm{L}}$ channel across the brain tissue (Fig. 3e-f). Axon fibers are enwrapped by myelin, a multilayered membranous sheath that serves as an electrical insulator for the 
axon to provide rapid conduction of action potentials. Myelin contains a high content of lipids made of large amounts of cholesterol and phospholipids (e.g. sphingomyelin), a very similar composition to the exosomes studied above. Zooming into an individual myelinated axon, lipids signal appeared as a hollow structure with a core axon and a myelin covering (Fig. 3g).

Overall, MAGNIFIERS can not only examine the ultrafine structures but also advance in revealing their chemical compositions, such as lipids and nucleic acids.

\section{High-resolution metabolic imaging of Huntingtin aggregates.}

Metabolic activities are the outcome of gene expression and relate to many dysfunctions and diseases. Bioorthogonal chemical imaging is a new paradigm which utilizes 'chemical bonds' as tiny vibrational tags for profiling small metabolites in cells and animals ${ }^{40-42}$. Different from bulky fluorophores which easily affect the function of the attached small metabolites, vibrational tags are minimally perturbative to normal biological functions. Among them, carbon-deuterium bonds are particularly effective with little perturbation, broad accessibility and residing in the bioorthogonal spectral window (where no endogenous biomolecules vibrate). Leveraging the power of small vibrational tags, MAGNIFIERS shall be able to gauge nanoscale features of metabolic activity.

Abnormal protein aggregation is associated with many neurodegenerative diseases. Aggregationprone polyglutamine (polyQ) protein is directly linked to Huntington's disease pathogenesis. Recent evidence suggests metabolic dysregulation in aggregates, which could be a potential target for future therapies $^{43}$. Being able to visualize fine structures of protein synthesis in small aggregates in the cellular environment could gain mechanistic insights and uncover therapeutic opportunities. While superresolution fluorescence imaging techniques have been extensively applied to study polyQ aggregates on resolving fine details of structures ${ }^{44,45}$, they have failed to provide information on metabolic activities of these aggregates. Using deuterium-labeled amino acids (D-AAs) ${ }^{41}$, here we demonstrate the application of MAGNIFIERS for metabolic imaging by studying protein synthesis of polyQ. After concurrently expressing polyQ proteins and labeling newly synthesized proteins with D-AAs in HeLa cells (Supplementary Fig. 8a), we fixed the cells, then gelled and linearly expanded them $\sim 3.4$ fold in each dimension (Fig. 4a). Formation of PolyQ aggregates with different patterns (such as large inclusion bodies (IBs), fibrils and branched clusters) were observed in both the cytosol and nucleus through imaging the $\mathrm{CH}_{3}$ peak at $2941 \mathrm{~cm}^{-1}$ or the CD peak at $2135 \mathrm{~cm}^{-1}$ (Fig. 4b-d). The improved spatial resolution is beneficial for resolving closely packed small clusters (Fig. 4c, inset) and the heterogeneous patterns of individual protein aggregates (Fig. 4d, inset).

Interestingly, the spatial distributions of new proteins (CD channel) and old proteins $\left(\mathrm{CH}_{3}\right.$ channel) were not always consistent. Quantitatively, the protein synthesis dynamics can be evaluated via the ratio of $\mathrm{CD}$ intensity over $\mathrm{CH}_{3}$ intensity (Fig. 4e-i). In a cell with multiple IBs located in the nucleus, we observed higher $\mathrm{CD} / \mathrm{CH}_{3}$ values in the core of both large (Fig. 4f) and small (Fig. 4g) IBs. This may suggest different phases of aggregates formation: the initial seeding of aggregates rely on active loading of misfolded polyQ and later aggregates expansion recruit not only misfolded polyQ but more other surrounding normal proteins. However, in cells with abundant aggregate formation and severely impaired cellular structures, we observed higher metabolic activities close to the boundary of some our aggregates (Fig. 4h), which may be generated through fragmentation of large aggregates. In addition, through labeling different time periods of polyQ expression, we can monitor the growth trajectory of aggregates. For example, with pulse-chase labeling using regular medium first and D-AAs replaced medium later, we 
found large clusters of aggregates are metabolically inactive in a long time period (Supplementary Fig. $8 b)$.

Overall, MAGNIFIERS platform can map metabolic activities of protein synthesis and turnover with sub-diffraction-limited spatial resolution of $\sim 100 \mathrm{~nm}$. We demonstrated its utility in the context of studying polyQ aggregates inside mammalian cells, which shows various nanoscale patterns of new protein synthesis. The observed features (core and boundary) of polyQ aggregates all have sub-diffractionlimited length scales. While we demonstrated with D-AAs here for studying protein synthesis, this principle can be applied to many other cases such as tracing lipid uptake with deuterium-labeled fatty acids and detecting DNA synthesis with 5-ethynyl-2'-deoxyuridine (EdU).

\section{Nanoscale Raman dye imaging}

Multiplexed super-resolution protein imaging in 3D tissues remains a technical challenge. Limited by the broad excitation and emission bandwidth of fluorescence, it is fundamentally difficult for regular super-resolution techniques to detect more than 4 targets. While researchers have demonstrated superresolution imaging of multiple targets using direct $\mathrm{STORM}^{46}$, gel embedding and expansion ${ }^{26,47}$, and DNA-PAINT ${ }^{48,49}$, they all relied on several rounds of staining, imaging, stripping and/or restaining. These cyclic methods could be artifact-prone, considering fine structures are vulnerable to non-linear histological changes among cycles and necessitate higher precision on image alignment. To overcome these technical difficulties, we plan to employ a one-shot multiplexing approach, by leveraging much narrower vibrational transitions.

Recently, we developed a novel multiplexing method of electronic pre-resonant SRS (epr-SRS) which detects electronically coupled vibrational modes in far-red absorbing dyes with sub-micromolar sensitivity. Probe-wise, a specially engineered MARS dye palette with $\pi$-conjugated triple bonds has been developed, which generates single and sharp peaks at the cell-silent region ${ }^{6,8}$. Epr-SRS enables Raman detection of interesting biomarkers ranging from proteins, transcripts, and enzyme activities ${ }^{6}$. 50 . In particular, immuno-eprSRS with MARS dye-conjugated antibodies has emerged as an encouraging platform for one-shot multiplexed protein imaging6, 11, bypassing technical troubles involved in cyclic techniques. Yet, it only works in a diffraction-limited paradigm.

We first studied the interplay between epr-SRS and MAGNIFY. Because the enzyme-free homogenization step enables post-expansion labeling (Fig. 1a), more epitopes may be revealed ${ }^{51}$, thus enhancing the labeling efficiency and overall signal size of immuno-eprSRS. We tested immuno-eprSRS in MAGNIFIERS using MARS2228 NHS ester conjugated secondary antibody (dye structure in Fig. 5a) to stain the expanded mouse brain section. However, we observed nonspecific staining backgrounds to varying degrees when the regular staining buffer of $1 \times$ PBS was utilized (Fig. 5b, Supplementary Fig. 9ab). Considering MARS dyes are positively charged and the gel matrix contains negatively charged carboxyl groups, we reasoned this non-specific staining might originate from an electrostatic effect. Hence, we changed the staining and washing buffers to a high-salt solution of $9 \times$ PBS (charge shielding effect) and supplemented with high-concentration Triton X-100 (10\%) which has been reported to prompt antibody binding ${ }^{52}$. Effectively, non-specific staining was substantially mitigated as we observed correct staining pattern of Synaptophysin labeled with MARS-dye conjugated secondary antibody in mouse brain (Fig. 5c, Supplementary Fig. 9c).

We next demonstrated the ability of MAGNIFIERS in visualizing nano-architecture of specific proteins with MARS dyes. By performing immuno-eprSRS of actinin 4 (ACTN4) in human kidney FFPE 
tissue with MARS dye conjugated secondary antibody, we observed ultrafine structures of the podocyte foot process by targeting the nitrile mode of MARS2228 (Fig. 5d, Supplementary Fig. 10a), consistent with standard immunofluorescence result ${ }^{21}$. The periodic pattern of the foot processes, which have a width around $200 \mathrm{~nm}$, was clearly visualized in the line plot (Fig. 5e, Supplementary Fig. 10a). Going beyond immunolabeling, other labeling approaches such as glycoprotein labeling with lectins (Fig. 5f) and panproteome staining ${ }^{32}$ with NHS ester or maleimide functionalized MARS probes (Fig. 5g, Supplementary Fig. 10b-d) are generally viable to MAGNIFIERS in a 3D fashion (Supplementary Videos 3-4). Of note, MARS dye staining and imaging does not compromise label-free imaging of endogenous biomolecules, as shown in Fig. 5f. Collectively, these results demonstrated the applicability of Raman dye imaging with high molecular specificity in MAGNIFIERS.

\section{One-shot highly multiplexed nanoscale imaging}

We next set out to showcase the one-shot highly-multiplexed nanoscale imaging capability of MAGNIFIERS in the mouse brain tissue section. To pursue better image contrast, we employ a simple but effective signal amplification strategy by using recurrent staining of MARS-conjugated secondary antibodies. In detail, as illustrated in Supplementary Fig. 11a, two different secondary antibodies, each of which binds to the other, will be applied alternatively after regular immunostaining process. Since postexpansion staining was utilized, the apparent size of the antibody is much smaller compared to the targeted structure in expanded samples. Therefore, recurrent staining rounds are unlikely to affect the interpretation on the shape and size of targeted proteins, as has been calibrated through standard immunofluorescence ${ }^{53}$. We tested this amplification method on mouse brain tissue via targeting synaptophysin, a protein found in synaptic vesicles and commonly used as a marker of pre-synaptic structures ${ }^{54}$. After additional 4-round recurrent staining of MARS2228-conjugated secondary antibodies, we observed substantial signal enhancement without noticeable background (Supplementary Fig. 11b-c). Quantitatively, epr-SRS signal of the skeletal $\mathrm{C}=\mathrm{C}$ mode of MARS2228 increased by 1.7-fold and 2.8-fold after 2-round and 4-round amplification, respectively (Supplementary Fig. 11d).

With this strategy, we acquired three epr-SRS channels of synaptophysin, $\alpha$-tubulin and postsynaptic density-95 (PSD95) each with additional 4-round staining in expanded mouse brain tissue. Fluorescence channels are also readily retained for nucleus (DAPI), microtubule associated protein 2 (MAP2) and Lycopersicon esculentum (tomato) lectin (LEL). Adding up the chemical imaging of $\mathrm{CH}_{\operatorname{Pr}}$ and $\mathrm{CH}_{\mathrm{L}}$ channels, we achieved a total of eight channels in volume in a single round (Fig. 6a), with an expansion of $\sim 4.5$ fold in each dimension (in $1 \times$ PBS, effective resolution of $\sim 73 \mathrm{~nm}$ ). Such a one-shot method bypasses the error-prone registration procedure and is particularly favorable for precise profiling of nanoarchitectures in thick volumes.

To showcase the nanoscale spatial precision, we focused on two synaptic marker proteins of synaptophysin (marker of synaptic vesicles) and PSD95. The post-ExM image explicitly revealed synaptophysin juxtaposed with PSD95 puncta (Fig. 6b-c, Supplementary Video 5). The separation distance between synaptophysin-PSD95 pairs were measured after the Gaussian fitting of the signal intensity along the line perpendicular to the synaptic cleft (Fig. 6d). The synaptophysin-PSD95 separation was measured as $118 \pm 52 \mathrm{~nm}$ (Fig. 6e, mean \pm s.d., $\mathrm{n}=119$ synapses) in MAGNIFIERS. Our result is close to the prior measurement of separations between postsynaptic densities and synaptic vesicles of around $150 \mathrm{~nm}$ using super-resolution fluorescence imaging ${ }^{54}$. To our best knowledge, vibrational imaging has not been applied to visualize individual synaptic features before. Overall, we expect MAGNIFIERS will 
provide a parallel and scalable way to interrogate nano-architectures in 3D spatial context with molecular specificity.

\section{Discussion}

SRS microscopy and ExM, two emerging techniques, have been synergistically integrated in MAGNIFIERS, providing niches that are complementary to existing SRS or ExM imaging alone. On one side, MAGNIFIERS offers a simple and general way to achieve super-resolution Raman imaging; on the other side, it complements the prevalent fluorescence-based nanoscopy. Leveraging the wide-ranging capabilities of SRS microscopy, we have demonstrated three classes of applications: label-free chemical imaging, metabolic imaging, and multiplexed imaging with nanoscale precision (Fig. 1a). For chemical imaging (Figs. 2-3), MAGNIFIERS provides label-free access to visualize proteins, lipids and DNA biomolecules with effective resolution up to $\sim 41 \mathrm{~nm}$. We have demonstrated this utility in varied biologically meaningful scenarios, such as visualization of the ultrastructure of basal bodies and investigation of the chemical composition of EVs in human lung organoids. We foresee that MAGNIFIERS will contribute single-vesicle level understanding of EVs in vivo. For metabolic imaging (Fig. 4), we studied protein synthesis and turnover on protein aggregates using deuterated amino acid labeling. In multiplexed imaging with Raman dyes (Figs. 5-6), MAGNIFIERS offers a one-shot optical approach to simultaneously map multiple targets in 3D in mouse brain sections. Finally, these applications are additive. For instance, chemical imaging is compatible with imaging of metabolic activities or postexpansion Raman dye imaging, providing a comprehensive view of the chemical content of the sample.

A recent technique called VISTA combines label-free SRS imaging of protein with a special ExM protocol named magnified analysis of the proteome (MAP) ${ }^{29,55}$. Compared to VISTA, MAGNIFIERS demonstrates the full potential of expansion SRS microscopy on a much broader range of biological specimens by leveraging the most recent advancement in $\mathrm{ExM}^{30}$. Foremost, unlike VISTA which requires the special acrylamide co-fixation and only reportedly works on mouse brain tissues ${ }^{26,29}$, MAGNIFIERS is not limited to soft tissue such as the brain and features general sample compatibility across a broad range of tissue types and fixation methods. We have shown that mechanically tough tissues such as FFPE human kidney can be robustly expanded and imaged by MAGNIFIERS, thus making it readily applicable to the vast archive of human specimens. Moreover, by virtue of more effective preservation of biomolecules (near 100\%) and tunable expansion level, our approach offers better detection sensitivity as compared to VISTA. This not only enables nanoscale label-free imaging of protein, nucleic acid, and lipids, but also allows us to open up nanoscale metabolic imaging and highly multiplexed Raman dye imaging, which generally have weaker signals than label-free applications. Lastly, MAGNIFIERS provides finer spatial resolution over VISTA by 1.6-fold through leveraging excellent reversible linear expansion of $\sim 7.2$-fold. In contrast, VISTA only allowed up to $\sim 4.5$-fold expansion. Altogether, MAGNIFIERS is currently the only technique that truly holds the value behind the concept of Expansion SRS microscopy: (1) true label-free nanoscale imaging of protein, nucleic acid, and lipids; (2) superresolution vibrational metabolic imaging; and (3) super-multiplexing capability. In contrast, VISTA only demonstrated part of (1) but none of (2) and (3).

Future improvements and opportunities reside in several fronts. First, the full expansion potential of the MAGNIFY gel has not been thoroughly utilized. To pursue higher effective resolution, femtosecond pulse excitation could be a direct way to enhance broadband SRS signals such as C-H and C-D bonds by an order of magnitude ${ }^{56}$. In this way, full expansion of $\sim 10$-fold in water will be viable for label-free protein imaging. Moreover, for Raman dye imaging, other DNA-based signal amplification techniques 
such as immuno-SABER ${ }^{47}$ and isHCR ${ }^{57}$ are expected to boost the signal more efficiently. Particularly, these amplification strategies will further increase the multiplexity with a practical goal of over 20 channels ${ }^{7}$. Second, specimens become optically transparent after expansion and thus MAGNIFIERS has promising applications for thick specimens of millimeter scales, considering successful precedents in integrating tissue clearing with label-free $\mathrm{SRS}^{58}$ or highly-multiplexed Raman dye imaging ${ }^{11}$. This would open exciting applications to probe interactions between ultrastructure with high content over a long distance, such as with neuronal tracing. Finally, while SRS may have low throughput for profiling largevolume specimens at high precision, the reversible and size-controllable features of the MAGNIFY gel provide good flexibility to combine large volume profiling and small region-of-interest interrogation in the same sample.

\section{References}

1. Freudiger CW, et al. Label-free biomedical imaging with high sensitivity by stimulated Raman scattering microscopy. Science 322, 1857 (2008).

2. Hu F, Shi L, Min W. Biological imaging of chemical bonds by stimulated Raman scattering microscopy. Nat Methods 16, 830-842 (2019).

3. Cheng J-X, Xie XS. Vibrational spectroscopic imaging of living systems: An emerging platform for biology and medicine. Science 350, aaa8870 (2015).

4. Wei L, Hu F, Chen Z, Shen Y, Zhang L, Min W. Live-cell bioorthogonal chemical Imaging: Stimulated Raman scattering microscopy of vibrational probes. Acc Chem Res 49, 1494-1502 (2016).

5. Wei L, et al. Imaging complex protein metabolism in live organisms by stimulated Raman scattering microscopy with isotope labeling. ACS Chem Biol 10, 901-908 (2015).

6. Wei L, et al. Super-multiplex vibrational imaging. Nature 544, 465-470 (2017).

7. $\mathrm{Hu} \mathrm{F}$, et al. Supermultiplexed optical imaging and barcoding with engineered polyynes. Nat Methods 15, 194-200 (2018).

8. Miao Y, Shi L, Hu F, Min W. Probe design for super-multiplexed vibrational imaging. Phys Biol 16, 041003 (2019).

9. Wei L, Min W. Electronic preresonance stimulated Raman scattering microscopy. J Phys Chem Lett 9, 4294-4301 (2018).

10. Miao Y, Qian N, Shi L, Hu F, Min W. 9-Cyanopyronin probe palette for super-multiplexed vibrational imaging. Nat Commun 12, 4518 (2021).

11. Shi L, et al. Highly-multiplexed volumetric mapping with Raman dye imaging and tissue clearing. Nat Biotechnol, https://doi.org/10.1038/s41587-021-01041-z, (2021).

12. Lu F-K, et al. Label-free DNA imaging in vivo with stimulated Raman scattering microscopy. Proc Natl Acad Sci USA 112, 11624 (2015).

13. Gasecka A, Daradich A, Dehez H, Piché M, Côté D. Resolution and contrast enhancement in coherent anti-Stokes Raman-scattering microscopy. Opt Lett 38, 4510-4513 (2013).

14. Silva WR, Graefe CT, Frontiera RR. Toward label-free super-resolution microscopy. ACS Photonics 3, 79-86 (2016).

15. Gong L, Zheng W, Ma Y, Huang Z. Higher-order coherent anti-Stokes Raman scattering microscopy realizes label-free super-resolution vibrational imaging. Nat Photonics 14, 115-122 (2020).

16. Gong L, Zheng W, Ma Y, Huang Z. Saturated stimulated-Raman-scattering microscopy for farfield superresolution vibrational imaging. Phys Rev Appl 11, 034041 (2019). 
17. Watanabe K, et al. Structured line illumination Raman microscopy. Nat Commun 6, 10095 (2015).

18. Olson AP, Ertsgaard CT, Elliott SN, Lindquist NC. Super-Resolution Chemical Imaging with Plasmonic Substrates. ACS Photonics 3, 329-336 (2016).

19. Wassie AT, Zhao Y, Boyden ES. Expansion microscopy: principles and uses in biological research. Nat Methods 16, 33-41 (2019).

20. Chen F, Tillberg PW, Boyden ES. Expansion microscopy. Science 347, 543 (2015).

21. Zhao Y, et al. Nanoscale imaging of clinical specimens using pathology-optimized expansion microscopy. Nat Biotechnol 35, 757-764 (2017).

22. Tillberg PW, et al. Protein-retention expansion microscopy of cells and tissues labeled using standard fluorescent proteins and antibodies. Nat Biotechnol 34, 987-992 (2016).

23. Chozinski TJ, et al. Expansion microscopy with conventional antibodies and fluorescent proteins. Nat Methods 13, 485-488 (2016).

24. Chen F, et al. Nanoscale imaging of RNA with expansion microscopy. Nat Methods 13, 679-684 (2016).

25. Truckenbrodt S, Maidorn M, Crzan D, Wildhagen H, Kabatas S, Rizzoli SO. X10 expansion microscopy enables 25-nm resolution on conventional microscopes. EMBO Rep 19, e45836 (2018).

26. $\mathrm{Ku} \mathrm{T}$, et al. Multiplexed and scalable super-resolution imaging of three-dimensional protein localization in size-adjustable tissues. Nat Biotechnol 34, 973-981 (2016).

27. Park J, et al. Epitope-preserving magnified analysis of proteome (eMAP). Sci Adv 7, eabf6589.

28. Park H-E, et al. Scalable and Isotropic Expansion of Tissues with Simply Tunable Expansion Ratio. Adv Sci 6, 1901673 (2019).

29. Qian C, Miao K, Lin L-E, Chen X, Du J, Wei L. Super-resolution label-free volumetric vibrational imaging. Nat Commun 12, 3648 (2021).

30. Klimas A, et al. Nanoscale imaging of biomolecules using molecule anchorable gel-enabled nanoscale in-situ fluorescence microscopy. Nat Portfolio, 10.21203/rs.3.rs-858006/v1, (2021).

31. Mao C, et al. Feature-rich covalent stains for super-resolution and cleared tissue fluorescence microscopy. Sci Adv 6, eaba4542 (2020).

32. M'Saad O, Bewersdorf J. Light microscopy of proteins in their ultrastructural context. Nat Commun 11, 3850 (2020).

33. Waldman M, et al. Adult Minimal-Change disease: Clinical characteristics, treatment, and outcomes. Clin J Am Soc Nephrol 2, 445 (2007).

34. Burke MC, et al. Chibby promotes ciliary vesicle formation and basal body docking during airway cell differentiation. J Cell Biol 207, 123-137 (2014).

35. van Niel G, D'Angelo G, Raposo G. Shedding light on the cell biology of extracellular vesicles. Nat Rev Mol Cell Biol 19, 213-228 (2018).

36. Skotland T, Sandvig K, Llorente A. Lipids in exosomes: Current knowledge and the way forward. Prog Lipid Res 66, 30-41 (2017).

37. Pathan M, et al. Vesiclepedia 2019: a compendium of RNA, proteins, lipids and metabolites in extracellular vesicles. Nucleic Acids Res 47, D516-D519 (2019).

38. Kalluri R, LeBleu VS. The biology, function, and biomedical applications of exosomes. Science 367, eaau6977 (2020).

39. Verweij FJ, et al. The power of imaging to understand extracellular vesicle biology in vivo. Nat Methods, 10.1038/s41592-021-01206-3, (2021). 
40. Yamakoshi H, et al. Alkyne-tag Raman imaging for visualization of mobile small molecules in live cells. J Am Chem Soc 134, 20681-20689 (2012).

41. Wei L, Yu Y, Shen Y, Wang MC, Min W. Vibrational imaging of newly synthesized proteins in live cells by stimulated Raman scattering microscopy. Proc Natl Acad Sci USA 110, 11226 (2013).

42. Shi L, et al. Mid-infrared metabolic imaging with vibrational probes. Nat Methods 17, 844-851 (2020).

43. Huan C, Wei-na C, Sunggoan J, Sarah R, Stuart M, Bronwen M. Metabolic dysfunction in Alzheimers disease and related neurodegenerative disorders. Curr Alzheimer Res 9, 5-17 (2012).

44. Lu M, et al. Live-cell super-resolution microscopy reveals a primary role for diffusion in polyglutamine-driven aggresome assembly. J Biol Chem 294, 257-268 (2019).

45. Sahl SJ, Weiss LE, Duim WC, Frydman J, Moerner WE. Cellular inclusion bodies of mutant Huntingtin exon 1 obscure small fibrillar aggregate species. Sci Rep 2, 895 (2012).

46. Klevanski M, Herrmannsdoerfer F, Sass S, Venkataramani V, Heilemann M, Kuner T. Automated highly multiplexed super-resolution imaging of protein nano-architecture in cells and tissues. Nat Commun 11, 1552 (2020).

47. Saka SK, et al. Immuno-SABER enables highly multiplexed and amplified protein imaging in tissues. Nat Biotechnol 37, 1080-1090 (2019).

48. Guo S-M, et al. Multiplexed and high-throughput neuronal fluorescence imaging with diffusible probes. Nat Commun 10, 4377 (2019).

49. Agasti SS, Wang Y, Schueder F, Sukumar A, Jungmann R, Yin P. DNA-barcoded labeling probes for highly multiplexed Exchange-PAINT imaging. Chem Sci 8, 3080-3091 (2017).

50. Fujioka H, Shou J, Kojima R, Urano Y, Ozeki Y, Kamiya M. Multicolor Activatable Raman Probes for Simultaneous Detection of Plural Enzyme Activities. J Am Chem Soc 142, 2070120707 (2020).

51. Sarkar D, et al. Expansion revealing: Decrowding proteins to unmask invisible brain nanostructures. bioRxiv, 10.1101/2020.08.29.273540, 2020.2008.2029.273540 (2020).

52. Susaki EA, et al. Versatile whole-organ/body staining and imaging based on electrolyte-gel properties of biological tissues. Nat Commun 11, 1982 (2020).

53. Cho Y, et al. FRACTAL: Signal amplification of immunofluorescence via cyclic staining of target molecules. Nanoscale 12, 23506-23513 (2020).

54. Sauerbeck AD, et al. SEQUIN Multiscale Imaging of Mammalian Central Synapses Reveals Loss of Synaptic Connectivity Resulting from Diffuse Traumatic Brain Injury. Neuron 107, $257-$ 273.e255 (2020).

55. Lin L-E, Miao K, Qian C, Wei L. High spatial-resolution imaging of label-free in vivo protein aggregates by VISTA. Analyst 146, 4135-4145 (2021).

56. Zhang D, Slipchenko MN, Cheng J-X. Highly sensitive vibrational imaging by femtosecond pulse stimulated Raman loss. J Phys Chem Lett 2, 1248-1253 (2011).

57. Lin R, et al. A hybridization-chain-reaction-based method for amplifying immunosignals. Nat Methods 15, 275-278 (2018).

58. Wei M, et al. Volumetric chemical imaging by clearing-enhanced stimulated Raman scattering microscopy. Proc Natl Acad Sci USA 116, 6608 (2019). 


\section{Acknowledgments}

We kindly thank Lingyan Shi for the assistance in animal experiments, thank Chenyi Mao and Gianluca Oyarzún for helpful discussions. W.M. acknowledges support from NIH (R01 GM128214, R01 GM132860 and R01 EB029523). Y.Z. acknowledges support from Carnegie Mellon University, DSF charitable foundations, U.S. Department of Defense DoD VR190139, NIH Director's New Innovator Award DP2 OD025926-01. X. R. acknowledges support from DSF charitable foundations and the Department of Biomedical Engineering at Carnegie Mellon University. P.W. acknowledges T32 predoctoral training grant (Biomechanics in Regenerative Medicine, BiRM) from the National Institute of Biomedical Imaging and Bioengineering of NIH.

\section{Author contributions:}

LS performed SRS imaging experiments and analyzed related data. AK, BRG, FF and YZ performed all expansion experiments and related characterizations. ZC contributed to image processing of multiplex SRS images. PW and XR prepared lung organoid samples. YM synthesized MARS probes. LS, YZ and WM conceived the concept and wrote the manuscript with input from all authors. YZ and WM supervised the project.

\section{Competing interests:}

The authors declare the following competing financial interest(s): YZ, AK and FF are inventors on several inventions related to ExM methods.

\section{Data and materials availability:}

All data supporting this work are available in the main text or the supplementary materials. All raw data are available from the corresponding authors upon request. 
a
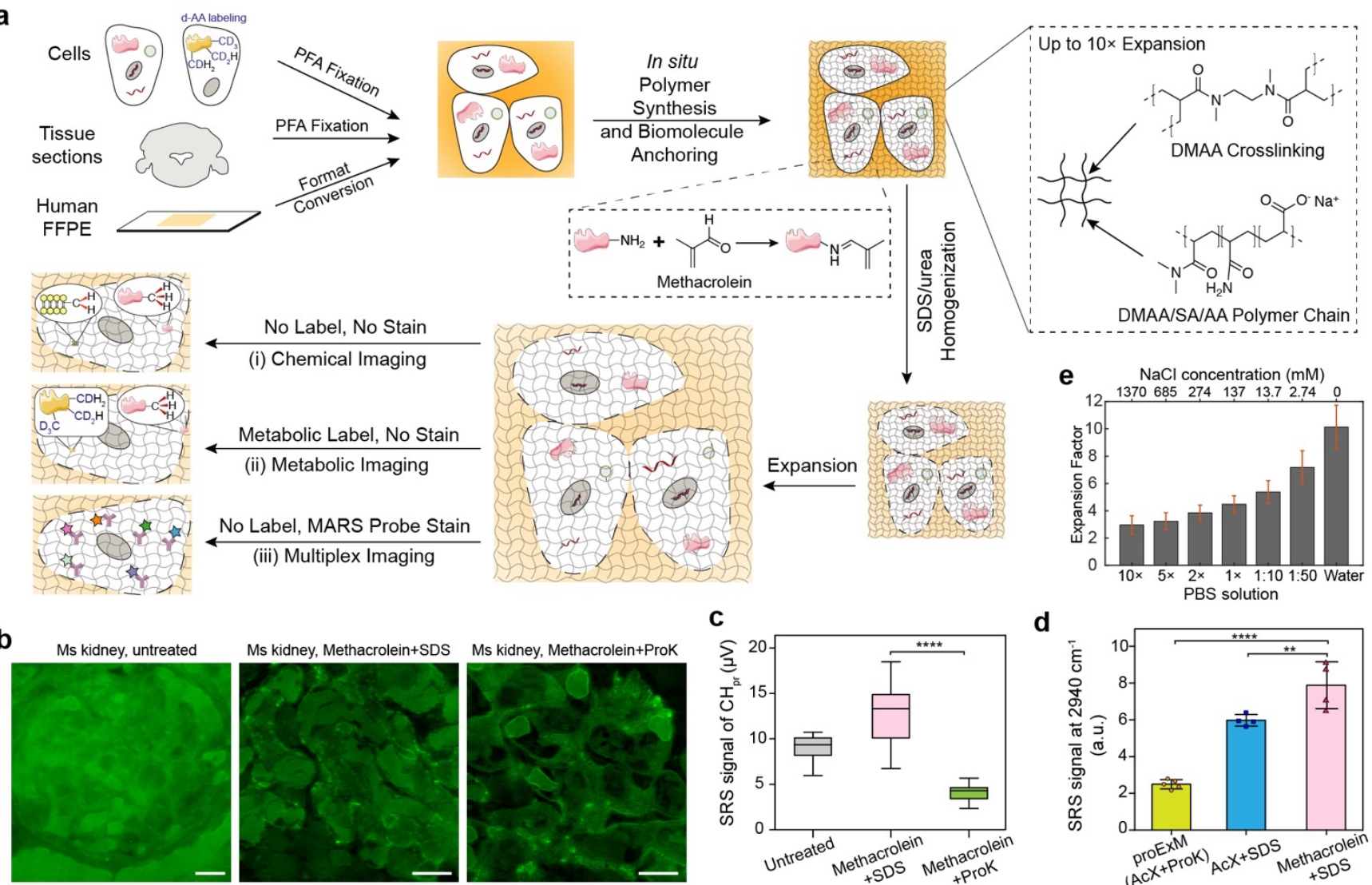

C

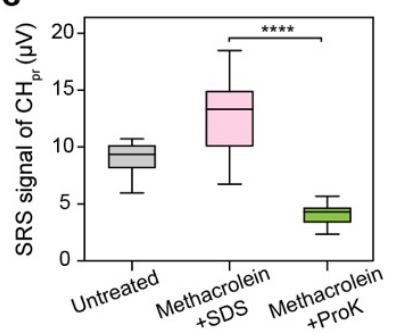

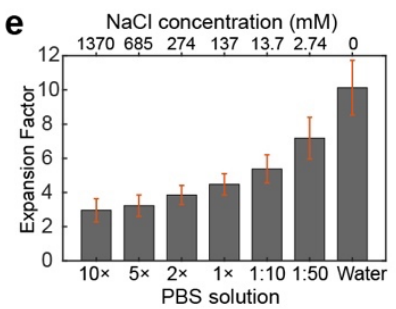
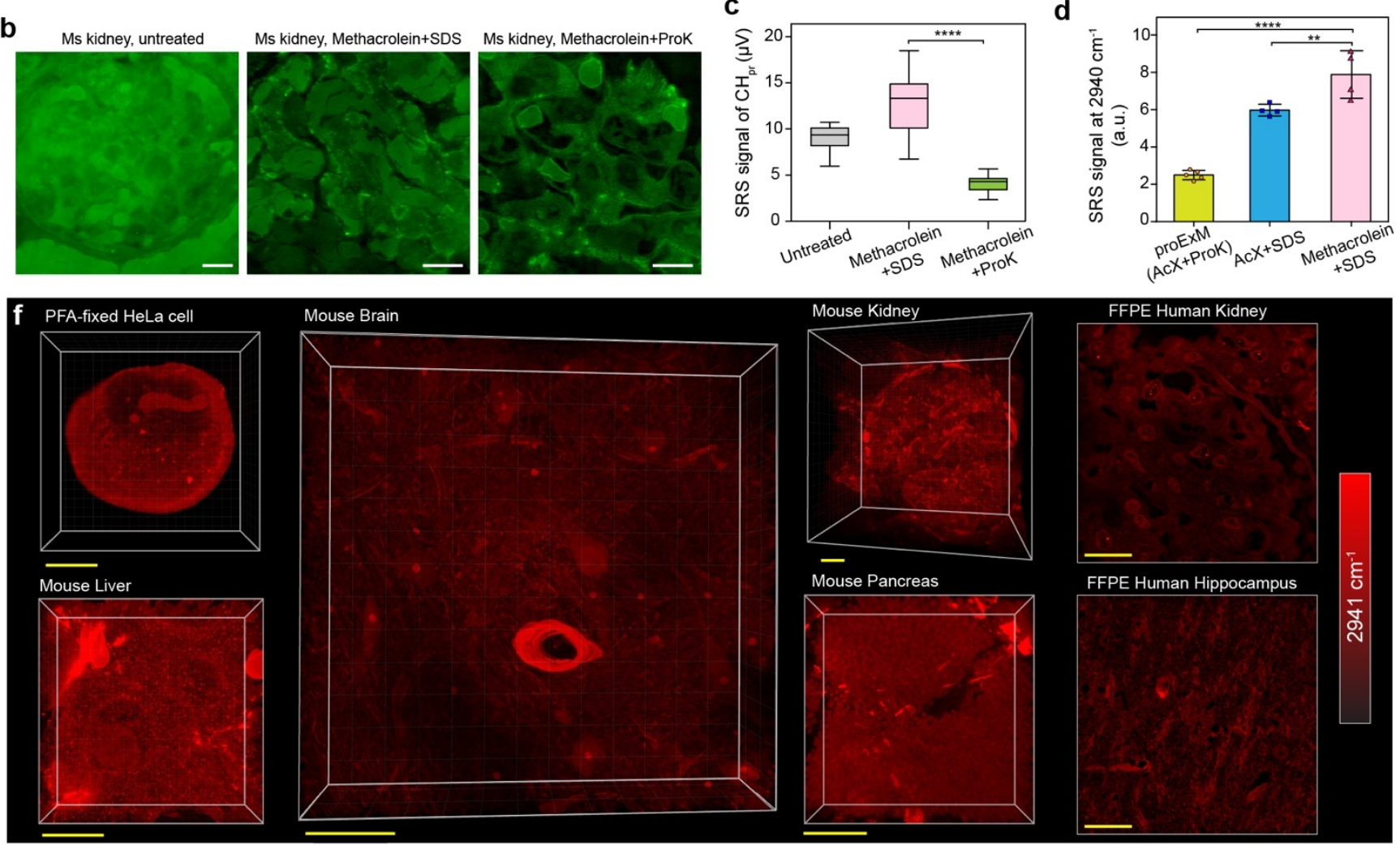

Mouse Liver

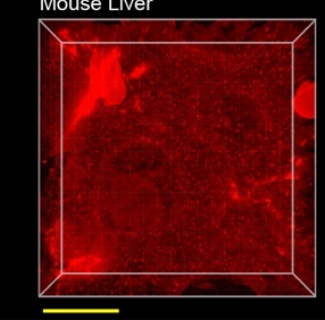

Fig. 1 Integration of SRS with an optimized ExM protocol (MAGNIFY). a, Schematics of the workflow of MAGNIFIERS and gel chemistry of MAGNIFY. During in situ polymer synthesis, methacrolein was added as part of the monomer solution to efficiently attach biomolecules to the growing matrix. The gel was formed from polymerization of cocktails of the monomers acrylamide (AA, nonionic monomer), sodium acrylate (SA, ionic monomer) and $N, N$-dimethylacrylamide (DMAA, nonionic self-cross-linker. Afterwards, the gel-embedded specimens were treated with sodium dodecyl sulfate (SDS)/urea solution in $80-90{ }^{\circ} \mathrm{C}$ for at least one hour for homogenization. PFA, 
paraformaldehyde. $\mathbf{b}$, SRS images of unmixed $\mathrm{CH}_{\text {protein }}$ signal of the glomerulus in the mouse kidney tissues. (left) untreated, (middle) expanded with methacrolein-linking and SDS/urea homogenization, (right) expanded with methacrolein-linking and Proteinase K digestion. c, Quantification of protein contents for untreated and expanded samples as shown in (b). SRS signals were plotted as mean \pm s.d. $(n=29,25,25$ regions of interest (ROIs)). In the box plot, the center indicates the median; the bottom and top edges of the box indicate the $25^{\text {th }}$ and $75^{\text {th }}$ percentiles, respectively; the whiskers extend to the minimum and maximum data points. Absolute signal in the untreated sample is converted with a volume dilution factor of $4.3^{3}=\sim 80$-fold after expansion. Two-tailed unpaired $t$-test, $* * * * P<0.0001, t=13$. d, Comparison of protein retention on the proExM protocol with the MAGNIFIERS protocol. SRS signals were plotted as mean \pm s.d. ( $=5,4,4$ ROIs). One-way ANOVA followed by Bonferroni's post hoc test using the 'Methacrolein+SDS' as the control column, $* * * * P<0.0001$ and $* * P=0.0085$. e, Measurement of expansion factors (mean \pm s.d.) in PBS buffers with different salt concentrations. Corresponding salt concentrations of used PBS buffer solution are labeled on the up X axis. Values of $\mathrm{n}$ are provided in Supplementary Table 2. f, 3D-rendered SRS images of $\mathrm{CH}_{3}$ peak at $2941 \mathrm{~cm}^{-1}$ of PFA-fixed HeLa cell, mouse brain, liver, kidney and pancreas tissues, and FFPE human kidney and brain hippocampus tissue. Yellow scale bars are not corrected for the expansion factor. Scale bars, $10 \mu \mathrm{m}$ in (b); $50 \mu \mathrm{m}$ (postexpansion) in (f). 

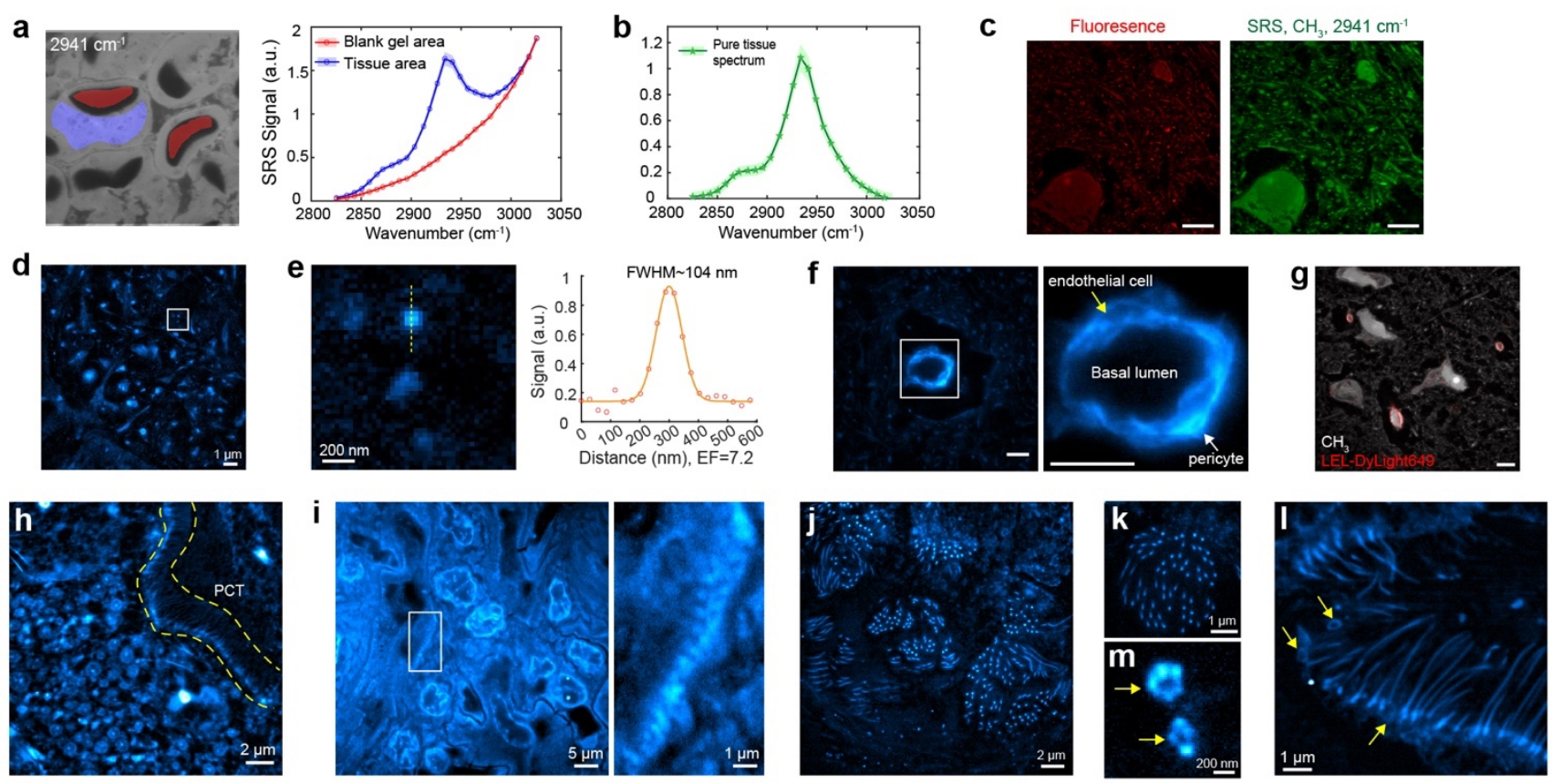

Fig. 2 MAGNIFIERS visualizes ultrafine structures of biological samples via label-free protein imaging. a, Hyperspectral SRS imaging of the $\mathrm{C}-\mathrm{H}$ stretching region on expanded FFPE human kidney. Left, masks of tissue area (red) and blank gel area (blue) for spectral analysis labeled on the SRS image of $2941 \mathrm{~cm}^{-1}$. Right, SRS spectra of two masked areas. Single-frequency SRS images are in Supplementary Fig. 4a. b, Background-removed SRS spectrum of expanded FFPE human kidney. c, SRS CH 3 image at $2941 \mathrm{~cm}^{-1}$ and fluorescence image of Alexa Fluor 555 NHS ester labeled mouse brain tissue. d, $\mathrm{CH}_{3}$ image of extended mouse brain in 1/50× PBS (7.2-fold expansion) with a $1.2 \mathrm{NA}$ objective. e, Zoom into the region outlined by the white box in (d). Right, line profile of a subdiffraction-limited spot outlined by the yellow line. f, Fine structures of blood-brain barrier in mouse brain tissue. Single endothelial cells and a pericyte were visualized. Images were collected using a 1.05 NA objective in $1 \times$ PBS (4.5-fold expansion). Right, zoom into the region outlined by the white box. $g$, Blood vessels and capillaries stand out in the protein channel, confirmed by lectin staining. Expanded mouse brain tissue labeled with Lycopersicon Esculentum lectin (LEL)-DyLight 649 and imaged with a 1.05 NA objective in $1 \times$ PBS (4.5-fold expansion). $\mathbf{h}$, SRS protein image in expanded mouse kidney. PCT, proximal convoluted tubule. Yellow region highlights the cilia on the surface of PCT. i, SRS protein image reveals periodic podocyte foot processes in expanded FFPE human kidney tissue. Right, zoom into the region outlined by the white box. Images were collected using a 1.2 NA objective in $1 \times$ PBS (2.3-fold expansion). $\mathbf{j}-\mathbf{m}$, Flower-like cilia structure ( $\mathbf{j}, \mathbf{k})$ and the ring structure at the base of cilia $(\mathbf{l}, \mathbf{m})$ in the expanded human lung organoid were visualized by the protein channel at $2941 \mathrm{~cm}^{-1}$.

Yellow arrows in $(\mathbf{k}, \mathbf{m})$ highlight the basal bodies. Images were collected with a 1.05 NA objective, $(\mathbf{j}$, l) in $1 \times$ PBS (4.5-fold expansion) and (k, m) in 1/50× PBS (7.2-fold expansion). Scale bars, $200 \mathrm{~nm}$ in $(\mathbf{e}, \mathbf{m}) ; 1 \mu \mathrm{m}$ in (d, i (right), $\mathbf{k}, \mathbf{l}) ; 2 \mu \mathrm{m}$ in (f, $\mathbf{h}, \mathbf{j}) ; 5 \mu \mathrm{m}$ in (c, $\mathbf{g}, \mathbf{i}$ (left)). 

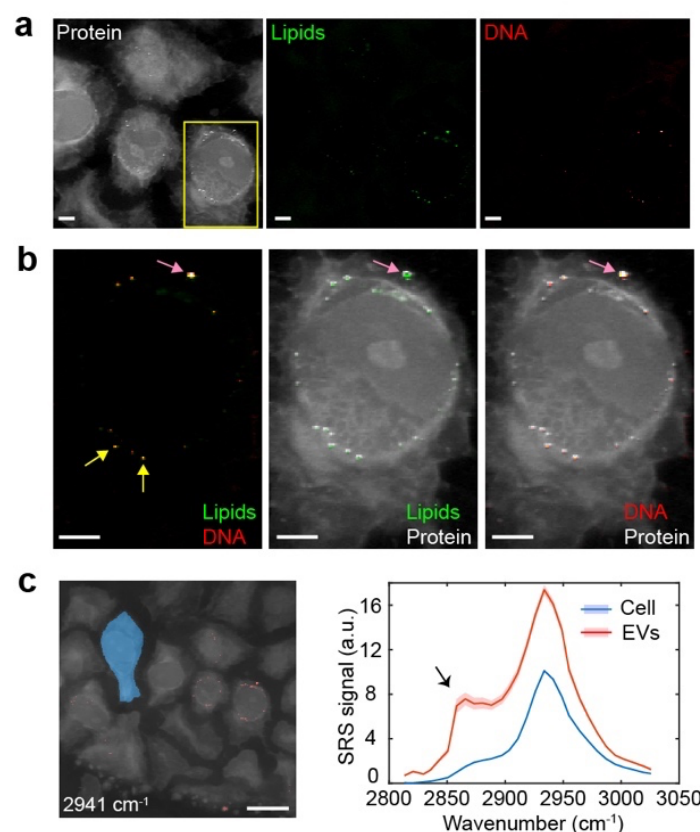

b
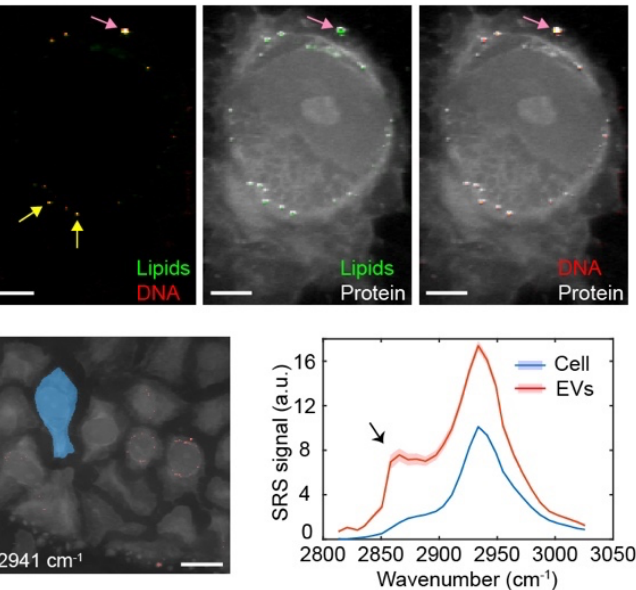

d
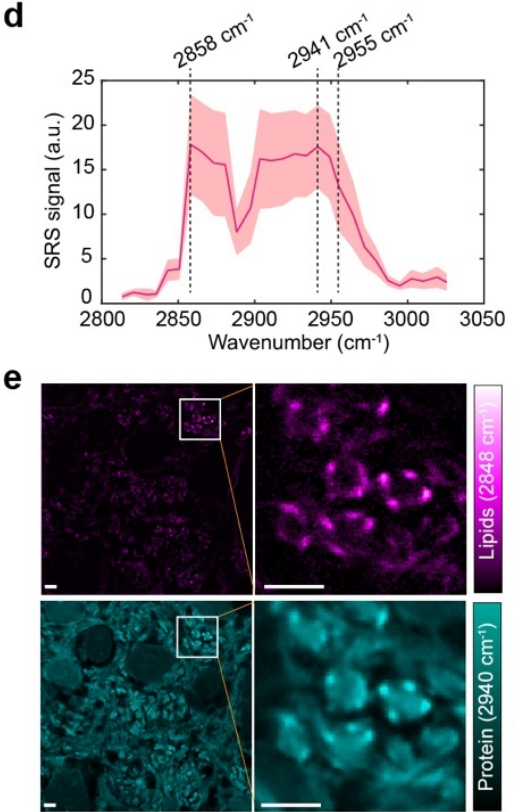

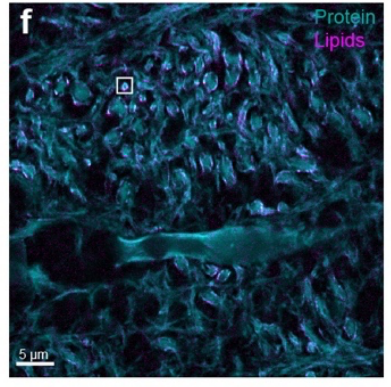

g
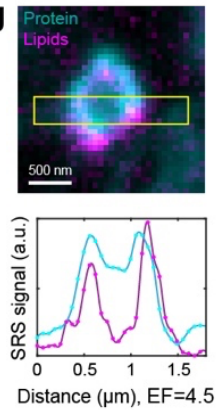

Fig. 3 Label-free nanoscale imaging of chemical compositions. a, Spectrally unmixed C-H channels for protein, lipids and DNA in expanded human lung organoid. b, Two-color overlayed images of zoomin areas outlined by the yellow box in (a). Arrows indicate EVs containing lipids and DNA. Images were collected using a 1.05 NA objective in $1 \times$ PBS (4.5-fold expansion). c, SRS spectral analysis of the cell area (blue) and extracellular vesicles (EVs, red) in the human lung organoid. Left, selected areas for spectral analysis representing the cell (blue) and EVs (red). Right, background-subtracted SRS spectra. Arrow indicated a side peak around $2865 \mathrm{~cm}^{-1}$ contributing from the $\mathrm{CH}_{2}$ signal of lipids in small EVs. Raw hyperspectral SRS spectra and all single-frequency SRS images are in Supplementary Fig. 6a. d, Hyperspectral SRS spectrum at $\mathrm{C}-\mathrm{H}$ region of a single EV in (b) marked by a pink arrow. e, Spectrally unmixed C-H channels for protein and lipids in the expanded mouse brain tissue. Right, zoom into areas outlined by the white boxes. Images were collected using a 1.05 NA objective in $1 \times$ PBS (2.0-fold expansion, original ExM gel). f, Overlayed image of protein and lipids in the expanded mouse brain tissue. Images were collected using a 1.05 NA objective in $1 / 25 \times$ PBS (2.6-fold expansion, original ExM gel). g, Magnified area outlined by the white box in (f) of transverse cross-section of an individual axon. Down, corresponding plot of the intensity of proteins and lipids along the yellow box long axis. Shaded areas in $(\mathbf{a}, \mathbf{c}, \mathbf{d})$ indicate the s.e.m. from different pixels. Scale bars, $500 \mathrm{~nm}$ in $(\mathbf{g}) ; 2$ $\mu \mathrm{m}$ in (a, b, e); $5 \mu \mathrm{m}$ in (f); $10 \mu \mathrm{m}$ in (c). 
a

$\mathrm{mH} t \mathrm{tt}(\mathrm{Q} 74)$-GFP plasmid transfection

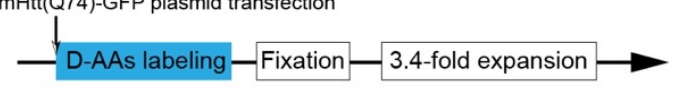

b

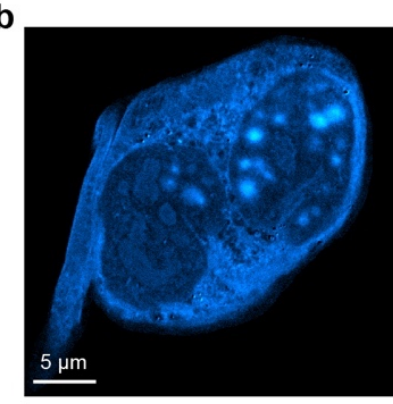

C

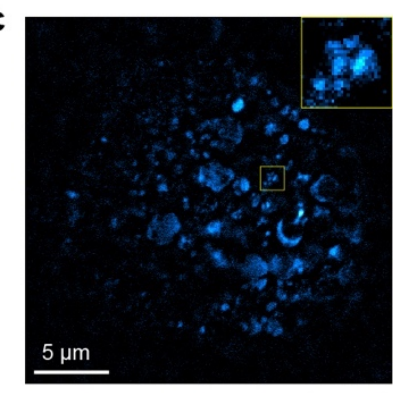

d

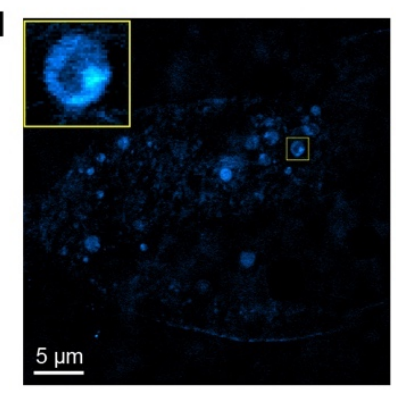

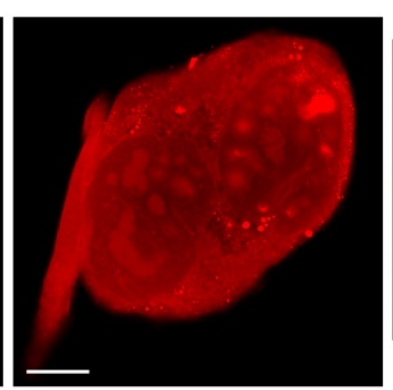
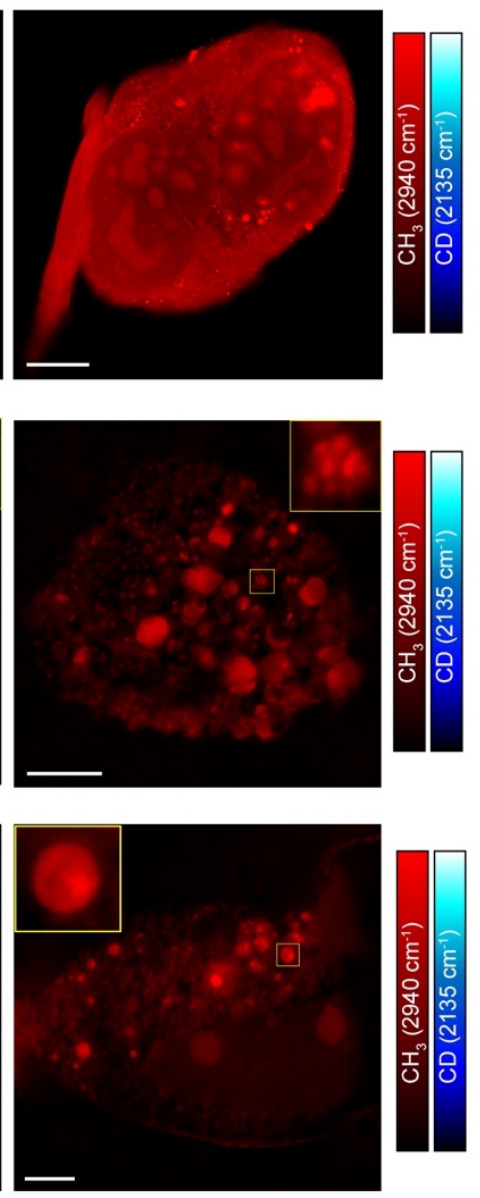

e
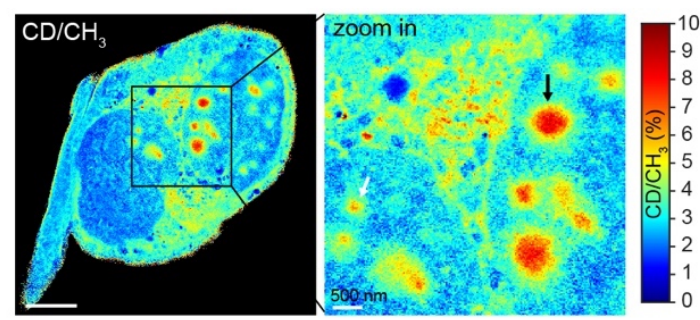

f

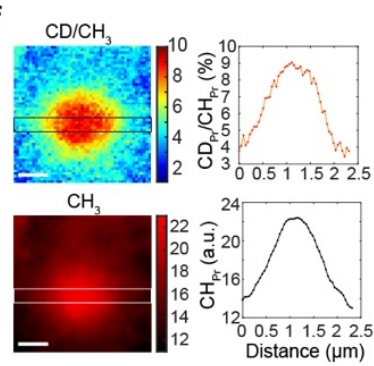

g $\mathrm{CD/CH}$ h
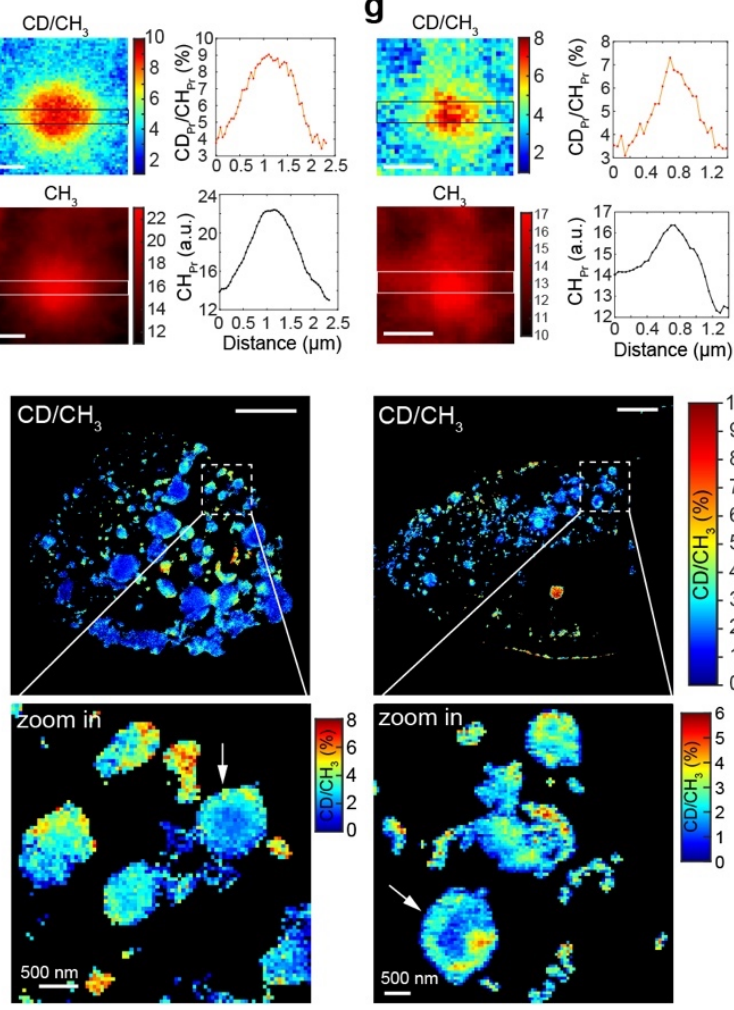

$\mathrm{CH}_{3}$
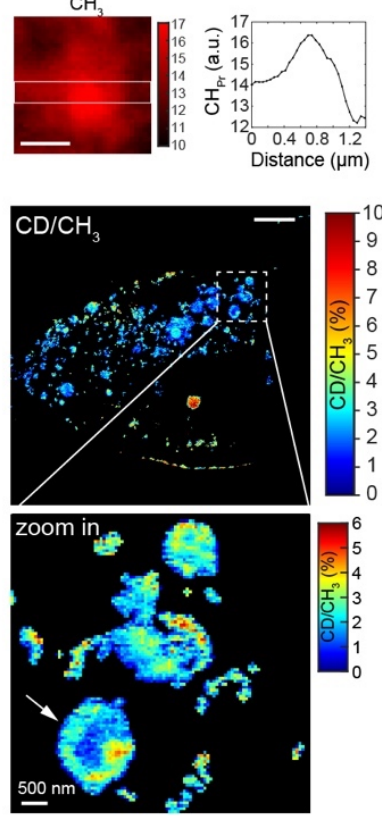

Fig. 4 Super-resolution metabolic imaging of newly synthesized protein in Huntingtin aggregates. a, Deuterated amino acids (D-AAs) labeling in time with simultaneous expression of mutant huntingtin (mHtt74Q-GFP) proteins for $48 \mathrm{hrs}$. The cartoons display the experimental pipeline of plasmid transfection, medium exchanges, fixation and expansion. b-d, Representative ROIs of $\mathrm{CH}_{3}$ images at $2941 \mathrm{~cm}^{-1}$ and CD images at $2135 \mathrm{~cm}^{-1}$ after expansion. Up in (c, d), zoom into the region outlined by the yellow box. e, Ratio images of $\mathrm{CD} / \mathrm{CH}_{3}$ for $\mathrm{ROI}$ in (b). Right, zoom into the region outlined by the black box. Arrow pointed out representative aggregates analyzed in (f-g). (f) Analysis of the aggregate pointed with black arrow in (e). g, Analysis of the aggregate pointed with white arrow in (e). h, Ratio images of $\mathrm{CD} / \mathrm{CH}_{3}$ for $\mathrm{ROI}$ in $(\mathbf{c}, \mathbf{d})$. Down, zoom into the regions outlined by the dotted box. All images were collected with a 1.05 NA objective in $5 \times$ PBS (3.44-fold expansion). Scale bars, $5 \mu \mathrm{m}$ in (b-d, e (left), h (up)); $500 \mathrm{~nm}$ in (f-g, e (right), h (down)). 
a

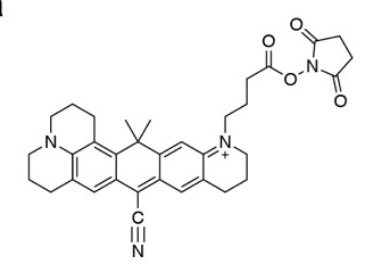

MARS2228 N-hydroxy-succinimidyl-ester

f

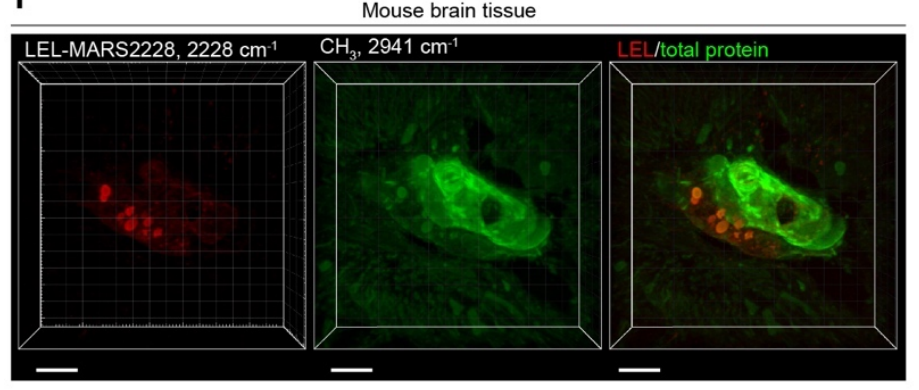

b

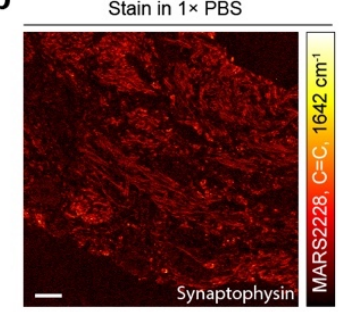

C

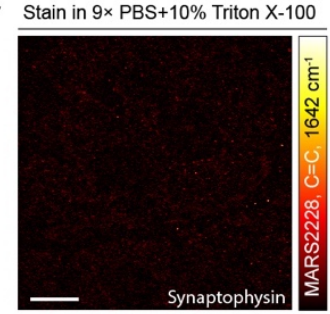

g Human lung organoid

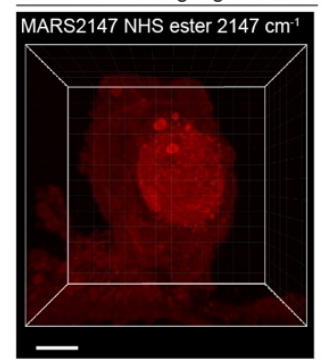

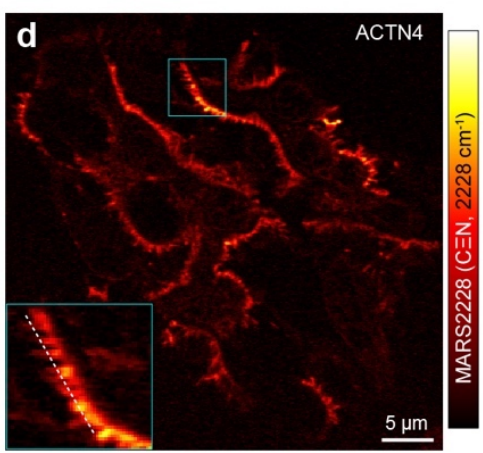

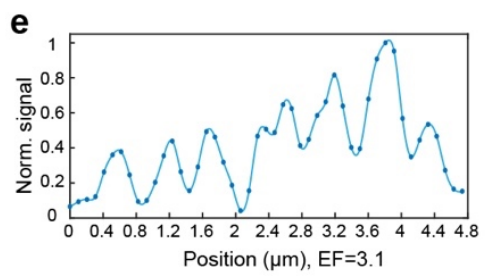

Fig. 5 Nanoscale Raman dye imaging. a, Chemical structure of NHS ester functionalized MARS2228. b, Staining background when $1 \times$ PBS was used as the staining buffer. c, Correct staining patterns of Synaptophysin using $9 \times$ PBS with $10 \%$ Triton-X as the staining buffer. d, Immuno-eprSRS image of actinin-4 (ACTN4, specifically label tertiary podocyte foot processes) in FFPE human kidney tissue with MARS2228. Inset, zoom into the region outlined by the blue box, dotted white curve within the inset indicates the line cut analyzed below. e, Normalized epr-SRS signal of the nitrile mode of MARS2228 along the line cut of the inset in (d). f, 3D-rendered epr-SRS image of MARS2228conjugated Lycopersicon Esculentum lectin (LEL) labeling and $\mathrm{CH}_{3}$ image of $2941 \mathrm{~cm}^{-1}$. g, 3D-rendered epr-SRS image of expanded human lung organoid stained with MARS2147 NHS ester dye. Images were collected using a 1.05 NA objective (d) in 1/50× PBS (3.1-fold expansion, original ExM gel), (f-g) in $1 \times$ PBS (4.5-fold expansion). Scale bars, $10 \mu \mathrm{m}$ in $(\mathbf{b}, \mathbf{c}) ; 5 \mu \mathrm{m}$ in $(\mathbf{d}, \mathbf{f}, \mathbf{g})$. 

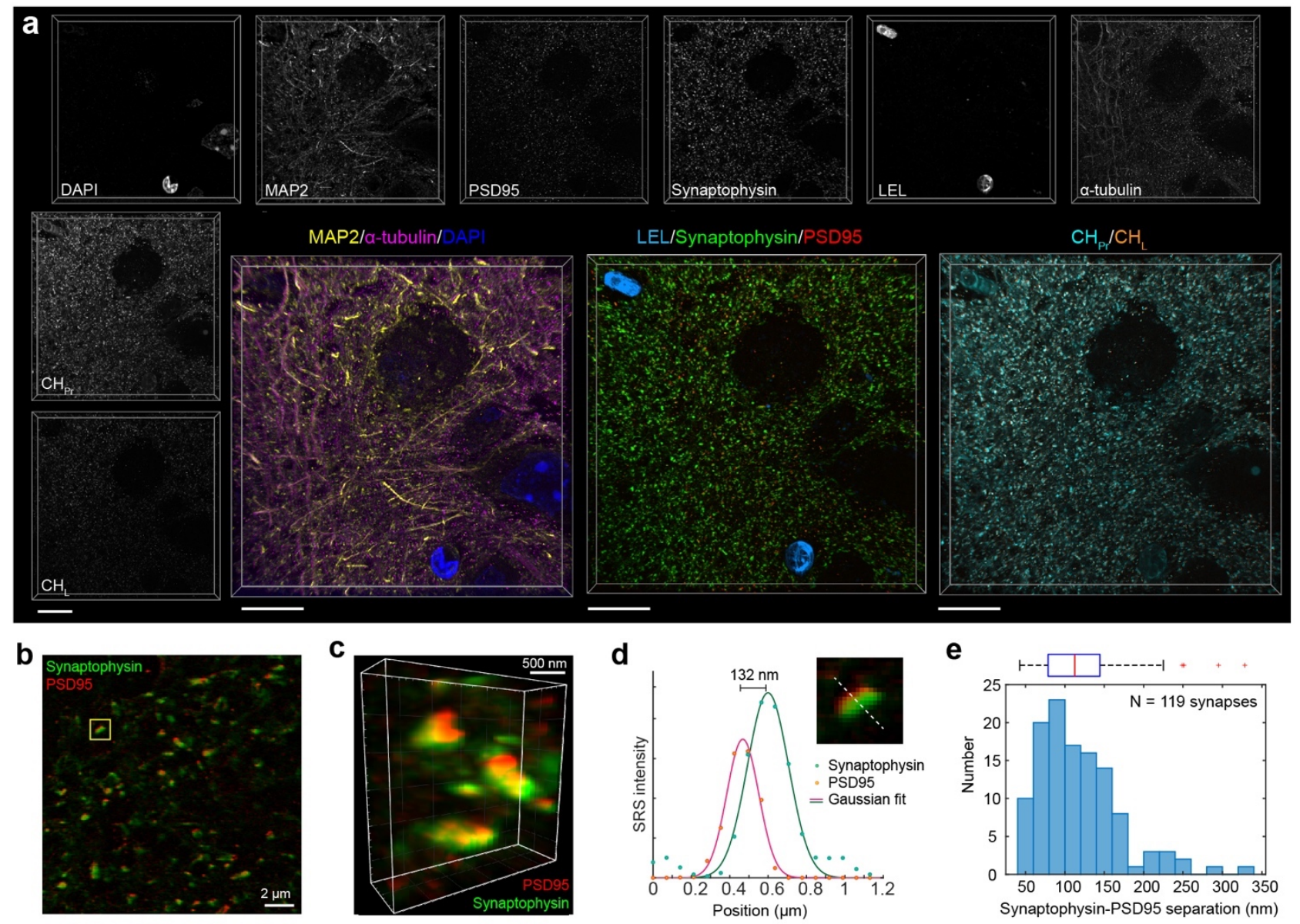

Fig. 6 One-shot highly multiplexed nanoscale imaging. a, One-shot 8-plex 3D-rendered nanoscale imaging of post-expansion mouse brain slice. Fluorescence: DAPI (total DNA), microtubule associated protein 2 (MAP2, Cy3), Lycopersicon Esculentum lectin (LEL-DyLight 649, blood vessels). SRS: Synaptophysin (MARS2228, synapse vesicles), $\alpha$-tubulin (MARS2176), postsynaptic density protein 95 (PSD95, MARS2147, post-synaptic membrane), $\mathrm{CH}_{\mathrm{Pr}}$ and $\mathrm{CH}_{\mathrm{L}}$. Voxel size is $0.318 \times 0.318 \times 1 \mu \mathrm{m}$ in post-expansion distance. b, Single-plane image of overlayed synaptophysin and PSD95 channels. $\mathbf{c}$, Zoom-in of 3D-rendered overlayed synaptophysin and PSD95 image. Yellow box outlined zoom-in image in (d) for separation distance analysis. $\mathbf{d}$, A representative image of single synapses and corresponding plot of the staining intensity of PSD95 and synaptophysin along the dotted line. Solid lines, Gaussian fits. e, Statistical analysis of Synaptophysin-PSD95 separations ( $\mathrm{N}=119$ synapses). Above is the box plot of the separation distances. In the box plot, the center indicates the median; the bottom and top edges of the box indicate the $25^{\text {th }}$ and $75^{\text {th }}$ percentiles, respectively; the whiskers extend to the minimum and maximum data points; the outliers are plotted individually using the '+' marker symbol. Images were acquired with a $25 \times$ objective in $1 \times$ PBS ( 4.5 -fold expansion). Scale bars, $10 \mu \mathrm{m}$ in (a), $2 \mu \mathrm{m}$ in (b), $500 \mathrm{~nm}$ in (c). 